This document is the unedited Author's version of a Submitted Work that was subsequently accepted for publication in Chemistry of Materials, copyright (c) American Chemical Society after peer review. To access the final edited and published work see: https://dx.doi.org/10.1021/acs. chemmater. 7b02522. 


\section{Remanence plots as a probe of spin disorder in magnetic nanoparticles}

Jose A. De Toro, ${ }^{* \dagger}$ Marianna Vasilakaki, ${ }^{\ddagger}$ Su Seong Lee, $₫$ Mikael S. Andersson, ${ }^{\S}$

Peter S. Normile, " Nader Yaacoub, ${ }^{\perp}$ Peyton Murray, \# Elena H. Sánchez, Pablo

Muñiz, $\|$ Davide Peddis, ${ }^{\circledR}$ Roland Mathieu, ${ }^{\S}$ Kai Liu,\# Julian Geshev, ${ }^{\triangle}$ Kalliopi

N. Trohidou, $\ddagger$ and Josep Nogués*, $\nabla, \dagger \dagger$

$\dagger$ †nstituto Regional de Investigación Científica Aplicada (IRICA) and Departamento de Física Aplicada, Universidad de Castilla-La Mancha, 13071 Ciudad Real, Spain

$\ddagger$ Institute of Nanoscience and Nanotechnology, NCSR "Demokritos", 15310 Aghia Paraskevi, Attiki,

Greece

【Institute of Bioengineering and Nanotechnology, 31 Biopolis Way, The Nanos, Singapore 138669, Singapore

§Department of Engineering Sciences, Uppsala University, Box 534, SE-751 21 Uppsala, Sweden

|| Instituto Regional de Investigación Científica Aplicada (IRICA) and Departamento de Física Aplicada,

Universidad de Castilla-La Mancha, 13071 Ciudad Real, Spain

$\perp$ Institut des Molécules et Mateŕiaux du Mans CNRS UMR-6283, Université du Maine, F-72085 Le Mans, France

\#Department of Physics, University of California, Davis, CA 95616, USA

@ Istituto di Struttura della Materia - CNR, 00015 Monterotondo Scalo (RM), Italy

$\triangle$ Instituto de Física, Universidade Federal do Rio Grande do Sul, 91501-970 Porto Alegre, RS, Brazil

$\nabla$ Catalan Institute of Nanoscience and Nanotechnology (ICN2), CSIC and The Barcelona Institute of

Science and Technology, Campus UAB, Bellaterra, 08193 Barcelona, Spain

††ICREA, Pg. Lluís Companys 23, 08010 Barcelona, Spain

E-mail: JoseAngel.Toro@uclm.es; Josep.Nogues@uab.cat 


\begin{abstract}
Remanence magnetization plots (e.g., Henkel or $\delta M$ plots) have been extensively used as a straightforward way to determine the presence and intensity of dipolar and exchange interactions in assemblies of magnetic nanoparticles or single domain grains. Their evaluation is particularly important in functional materials whose performance is strongly affected by the intensity of interparticle interactions, such as patterned recording media and nanostructured permanent magnets, as well as in applications such as hyperthermia and magnetic resonance imaging. Here we demonstrate that $\delta M$ plots may be misleading when the nanoparticles do not have a homogeneous internal magnetic configuration. Substantial dips in the $\delta M$ plots of $\gamma-\mathrm{Fe}_{2} \mathrm{O}_{3}$ nanoparticles isolated by thick $\mathrm{SiO}_{2}$ shells indicate the presence of demagnetizing interactions, usually identified as dipolar interactions. Our results, however, demonstrate that it is the inhomogeneous spin structure of the nanoparticles, as most clearly evidenced by Mössbauer measurements, that has a pronounced effect on the $\delta M$ plots, leading to features remarkably similar to those produced by dipolar interactions. X-ray diffraction results combined with magnetic characterization indicate that this inhomogeneity is due to the presence of surface structural (and spin) disorder. Monte Carlo simulations unambiguously corroborate the critical role of the internal magnetic structure in the $\delta M$ plots. Our findings constitute a cautionary tale on the widespread use of remanence plots to assess interparticle interactions, as well as offer new perspectives in the use of Henkel- and $\delta$ M-plots to quantify the rather elusive inhomogeneous magnetizations states in nanoparticles.
\end{abstract}

\title{
Introduction
}

Exchange and dipolar interactions between grains or particles are essential to understanding the behavior of magnetic polycrystalline and colloidal materials. ${ }^{1,2}$ Indeed, these interactions are key to the performance of many common magnetic materials, e.g., permanent magnets, ${ }^{3,4}$ magnetic recording media, ${ }^{5,6}$ magnetically soft materials for high frequency ap- 
plications ${ }^{7}$ where dipolar interactions may have undesirable effects, such as aggregation of nanoparticles in biomedical applications. ${ }^{8-10}$ Magnetic interactions control the properties of sufficiently dense assemblies of magnetic nanoparticles and nanostructures, tailoring their functional properties, e.g., blocking (or freezing) temperature, coercivity, remanent magnetization, switching-field distribution and effective anisotropy, among others. ${ }^{11-16}$ In fact, interactions are the basis of a large number of nanoparticle-based magnetic materials, e.g., superferromagnets, superspin glasses, artificial spin ice, long range self-assemblies, or ferrofluids. ${ }^{15,17-21}$ Given the crucial importance of interactions in magnetic nanostructures, many direct and indirect approaches have been used to try to quantify them: first order reversal curve (FORC) analysis, ${ }^{22,23}$ small angle neutron scattering, SANS, ${ }^{24-27}$ electron holography, ${ }^{28,29}$ magnetic force microscopy, ${ }^{30,31}$ Lorentz microscopy, ${ }^{32}$ Brillouin light scattering, ${ }^{33}$ resonant magnetic x-ray scattering ${ }^{34}$ and so on. However, one of the most accepted methods to assess interactions is the remanence plots technique (i.e., Henkel or $\delta M$ plots), ${ }^{35-37}$ which is routinely used to evaluate interactions between nanoparticles or grains ${ }^{38-55}$ both in fundamental studies ${ }^{56,57}$ and in diverse nanoparticle-based applications (e.g., patterned recording media, permanent magnets, or magnetic resonance imaging $\left.{ }^{11,38,39,55}\right)$. In particular, the $\delta M$ technique has been used to evaluate dipolar interaction in biomedical applications, where, for example, they have been shown to be crucial for the heating performance of nanoparticles in hyperthermia therapy. ${ }^{58-61}$ Interestingly, this approach is also widely used in paleomagnetism and rock magnetism ${ }^{62,63}$ or even biomagnetic systems. ${ }^{64,65}$ The technique is based on the fact that, for coherent rotation of non-interacting single-domain particles with uniaxial anisotropy, the isothermal remanent magnetization (IRM, $M_{r}$ ) and the direct current demagnetization remanence curve $\left(\mathrm{DCD}, M_{d}\right)$ are connected via the Wohlfarth relation: ${ }^{66}$ $M_{d}(H)=M_{r s}-2 M_{r}(H)$, where $M_{r s}$ is the saturation remanence and $H$ is the applied magnetic field [see Supporting Information for a more detailed explanation of the different terms]. Henkel observed that plots of $M_{d}(H)$ vs. $M_{r}(H)$ in heterogeneous alloys may present positive or negative curvatures deviating from the expected linearity. ${ }^{37}$ 


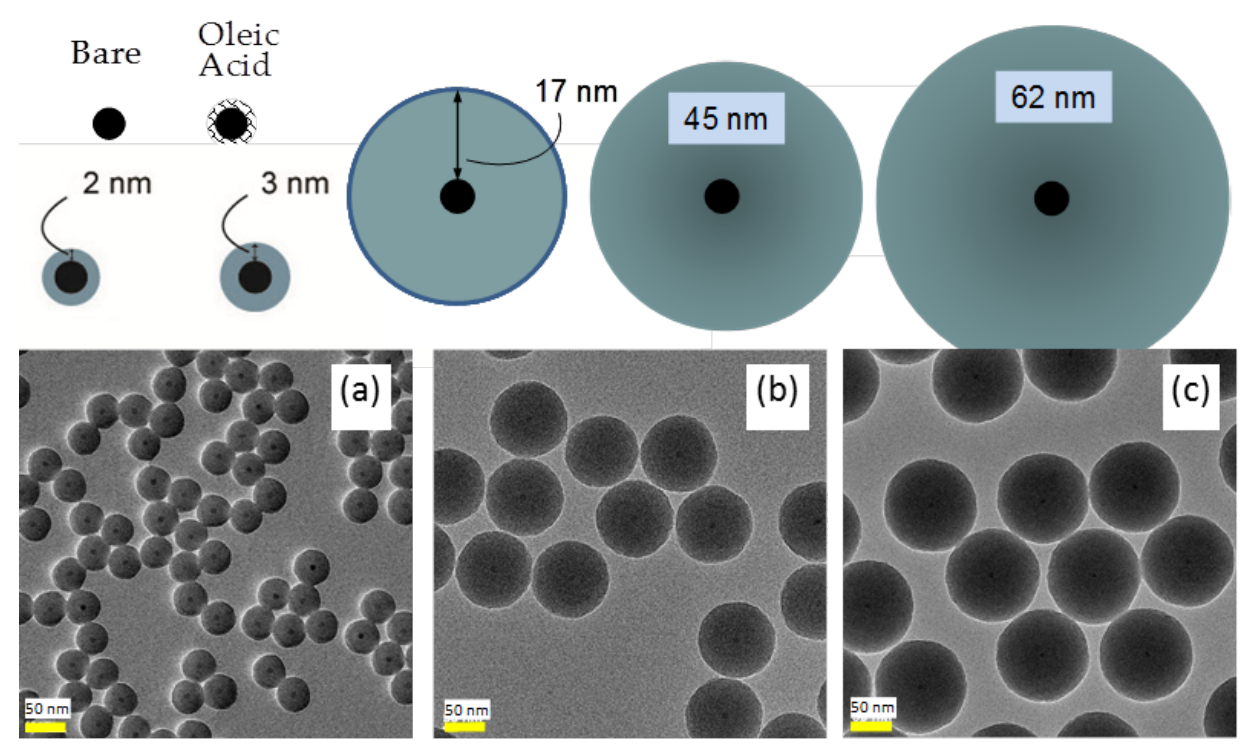

Figure 1: Top: schematic representation of the VSTx sample series with varying thickness of a non-magnetic $\mathrm{SiO}_{2}$ shell. Bottom: TEM images of the $8 \mathrm{~nm}$ nanoparticles with thickest $\mathrm{SiO}_{2}$ shells: (a) $t_{\mathrm{SiO}_{2}}=17 \mathrm{~nm}(\mathrm{VST} 17)$; (b) $t_{\mathrm{SiO}_{2}}=42 \mathrm{~nm}(\mathrm{VST} 42)$ and (c) $t_{\mathrm{SiO}_{2}}=62 \mathrm{~nm}$ (VST62).

In the 80's Kelly et al. introduced the concept of $\delta M$ plot, which measures the deviation from linearity in Henkel plots, conveniently preserving the explicit dependence on $H$, $\delta M(H)=M_{d}(H)-\left[M_{r s}-2 M_{r}(H)\right]$. Interparticle interactions are detected through the appearance of a negative dip (demagnetizing interactions; typically dipolar interactions) or a positive peak (magnetizing, usually exchange, interactions) in the $\delta M$ plots, while $\delta M(H)$ $=0$ has generally been taken as an indication of the absence of interactions. ${ }^{36,67}$

It is worth noting that the $\delta M$ plots for systems of non-interacting particles with cubic anisotropy are intrinsically positive ${ }^{68}$ and their shape may vary when different anisotropies co-exist. ${ }^{69}$ However, even in the uniaxial anisotropy case, the fact that magnetic single-phase and core/shell nanoparticles (particularly oxide nanoparticles) are usually not monodomains (i.e., cannot be simplified to a system of macro-spins), often exhibiting rather complex internal spin structures, ${ }^{70-74}$ may cast some doubts over the validity of the remanence curves approach for the evaluation of dipolar interactions. ${ }^{44-54,75-77}$

Here we investigate the interactions in $\gamma-\mathrm{Fe}_{2} \mathrm{O}_{3}$ nanoparticles coated by thick $\mathrm{SiO}_{2}$ shells (up to $62 \mathrm{~nm}$ ) via $\delta M$ plots and FORC. The $\delta M$ plots show clear negative dips, apparently 
implying dipolar interactions, even in the extremely magnetically dilute cases. Our results indicate, however, that while isolated nanoparticles with no spin canting have $\delta M=0$ and show no exchange bias, nanoparticles with surface spin canting display large negative $\delta M$ and finite bias. Thus, the inhomogeneous spin structure of the nanoparticles, even if only a thin disordered layer, can have a strong effect on the $\delta M$ plot, yielding features very similar to those observed for dipolar interactions. A systematic Monte Carlo study strongly supports the ordered core/ disordered surface magnetic structure as the origin of the unexpected features in the $\delta M$ plots.

\section{Results and discussion}

\section{Magnetic characterization}

To evaluate the strength of interactions as a function of the maghemite concentration, we carried out a systematic $\delta M$-plot study across two different series of $\gamma-\mathrm{Fe}_{2} \mathrm{O}_{3}$ nanoparticles: (i) fixed core diameter $\left(d_{T E M}=8 \mathrm{~nm}\right)$ with a varying- $\mathrm{SiO}_{2}$ shell-thickness series (VSTx, where $\mathrm{x}$ indicates the shell thickness in nm; see Fig. 1) and (ii) a changing-core-diameter (6-11 nm) series with a fixed $\mathrm{SiO}_{2}$ shell thick enough $(\approx 17 \mathrm{~nm})$ to guarantee the magnetic isolation of the maghemite cores (VCDx, where $\mathrm{x}$ indicates the approximate core diameter in $\mathrm{nm}$ ) (see Methods and Supporting Information). ${ }^{15,78,79}$ In all cases, X-ray diffraction and Mössbauer spectroscopy (see Supporting Information) consistently confirmed that the Fe oxide core is maghemite $\left(\gamma-\mathrm{Fe}_{2} \mathrm{O}_{3}\right)$. The same result was previously found for the $8 \mathrm{~nm}$ particles using Raman spectroscopy. ${ }^{80}$

The temperature dependence of the zero-field-cooled (ZFC) magnetization of all samples shows a peak at low temperatures signalling superparamagnetic (dilute systems) or freezing (dense systems) transitions ${ }^{15,79}$ (see Fig. S1a).

Figure 2 shows the normalized (to $\left.M_{r s}\right) M_{d}(H), M_{r}(H)$ and the resulting $\delta M(H)$ curves measured at $5 \mathrm{~K}$ for the VST0 (bare $8 \mathrm{~nm} \gamma-\mathrm{Fe}_{2} \mathrm{O}_{3}$ nanoparticles) and VST17 $\left(8 \mathrm{~nm} \gamma-\mathrm{Fe}_{2} \mathrm{O}_{3}\right.$ 


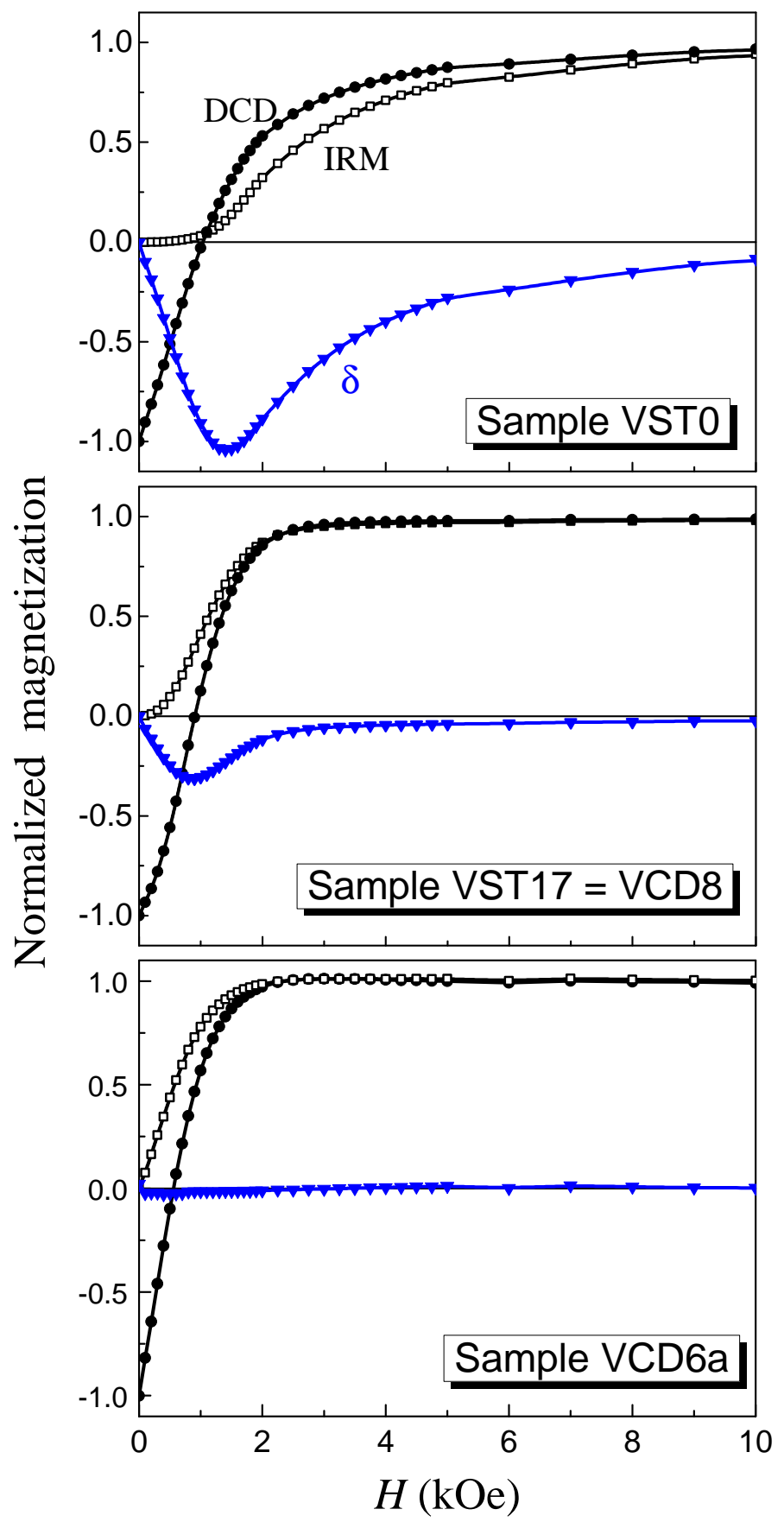

Figure 2: Normalized (to $M_{r s}$ ) isothermal remanence (IRM), dc demagnetization remanence (DCD), and the resulting $\delta M$ curves measured at $5 \mathrm{~K}$ in a dense assembly of maghemite particles $8 \mathrm{~nm}$ in diameter (upper panel) and in highly dilute assemblies of the same particles (middle panel) and of $6.2 \mathrm{~nm}$ nanoparticles (lower panel). 
nanoparticles with a $t_{\mathrm{SiO}_{2}}=17 \mathrm{~nm}$ shell) samples, as well as for the VCD6a system (with a similarly thick silica shell coating $6 \mathrm{~nm} \gamma-\mathrm{Fe}_{2} \mathrm{O}_{3}$ cores). The VST0 sample presents a very strong $\delta M \operatorname{dip}(\approx 108 \%)$, which indicates intense dipolar interactions. This is expected given that the absence of any protecting shell leaves the particles virtually in contact with each other, leading to an ideal superspin glass behavior ${ }^{81-83}$ characterized, e.g., by a very high freezing temperature (compared to that of isolated nanoparticles) and by shapes of the FC-ZFC curves closely reminiscent to those of atomic spin glasses, confirming the presence of strong interparticle interactions in this sample. Note that such strong $\delta M \operatorname{dips}$ have already (though rarely) been reported in other strongly interacting systems. ${ }^{84,85}$

Remarkably, a clear dip $(\approx 30 \%)$ in the $\delta M$ plot is also observed in sample VST17. As mentioned, negative $\delta M$ values are customarily taken to indicate the presence of demagnetizing dipolar interactions. However, the thick $\mathrm{SiO}_{2}$ shell ensures a large intercore distance yielding an estimated nearest-neighbour dipolar interaction strength, $T_{d d}\left[T_{d d}=\right.$ $\mu_{0} \mu^{2} /\left(4 \pi d^{3} k_{B}\right)$, where $\mathrm{d}$ is the average distance between particles and $\mu$ their magnetic moment $]^{86}$ below $1 \mathrm{~K}$. Therefore, dipolar interactions are negligible in the entire temperature range studied in this work. ${ }^{86}$ We can rule out the possible presence of multicore nanoparticles (i.e., the $\mathrm{SiO}_{2}$ does not coat single $\gamma-\mathrm{Fe}_{2} \mathrm{O}_{3}$ particles but aggregates of particles instead), previously observed in other systems prepared by similar methods, ${ }^{87,88}$ as accounting for the observed $\delta M$ dip: the synthesis procedure was optimized to avoid aggregation as confirmed by a thorough transmission electron microscopy (TEM) study (Fig. 1), examining thousands of particles. Importantly, the magnetic measurements also confirm that the thick $\mathrm{SiO}_{2}$ shell effectively suppresses interparticle interactions. For the VSTx series, the dependence of the ZFC peak temperature, $T_{B}$, on the capping layer thickness indicates that, for sufficiently large $t_{\mathrm{SiO}_{2}}, T_{B}$ becomes constant (Fig. 3, right axis). This is usually taken to imply negligible dipolar interactions at such great interparticle distances. This is corroborated by the shape of the FC curves, which become flat below $T_{B}$ for interacting particles, while for non-interacting particles they show a monotonic increase below $T_{B}$ (Fig. S1a). Finally, the 
classical test of $H / T$ scaling (Langevin behavior) was also verified in selected samples with thick silica shells after subtracting a high-field linear dependence (Fig. S1b). In fact, the FC and ZFC magnetization curves (Fig. S1a), the hysteresis loop at $300 \mathrm{~K}$ and the ac susceptibility curves measured at different frequencies were simultaneously fitted to an isolated particle model in a consistent way. ${ }^{15}$ Additionally, the magnetic volume obtained from a Langevin fit is slightly smaller than the physical volume, again discarding interactions ${ }^{89}$ (see Supporting Information).

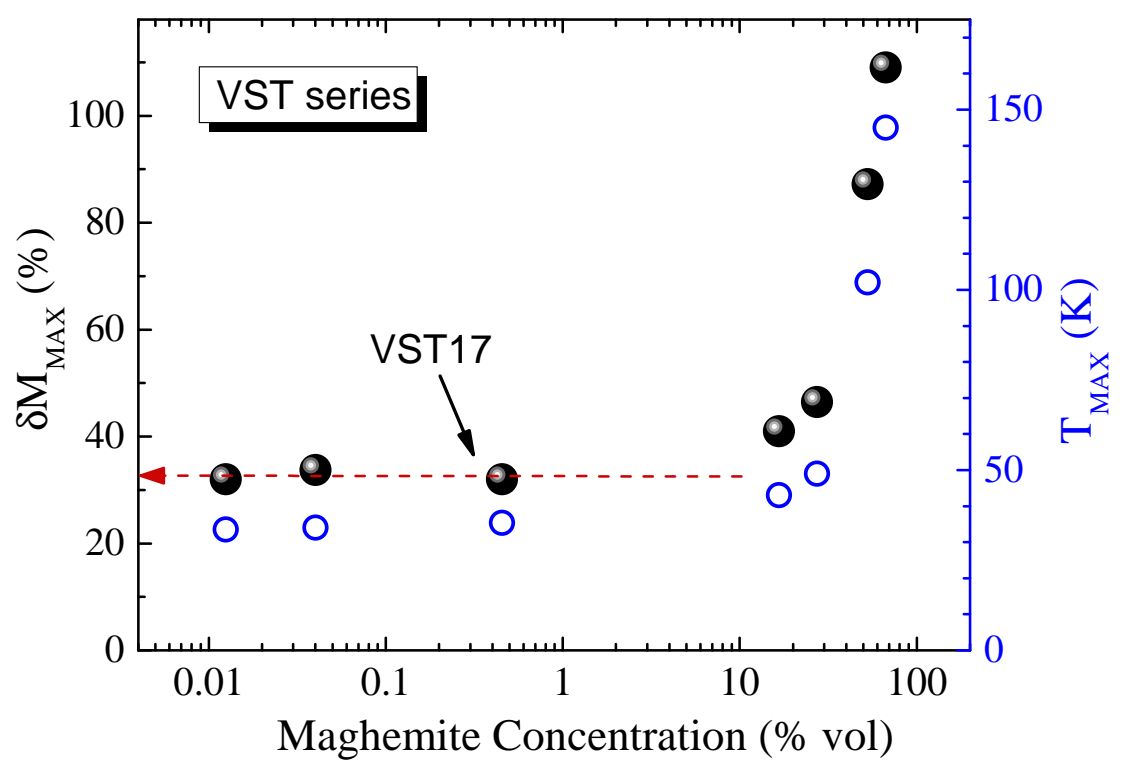

Figure 3: Maghemite concentration dependence of the normalized (to $M_{r s}$ ) depth of the $\delta M$ dip (left axis, where the dotted arrow indicates a background value for low concentrations) and of the peak temperature of the ZFC magnetization, $T_{M A X}$ (rights axis; see Fig. S1a).

An emphatic proof of the absence of any significant dipolar interactions is also provided by the observation of equivalent $\delta M$ behavior (and overall magnetic properties) in samples with even larger $t_{\mathrm{SiO}_{2}}$ in the VSTx series $\left(t_{\mathrm{SiO}_{2}}=45\right.$ and $62 \mathrm{~nm}$, both well below $0.1 \%$ volume concentration of $\gamma-\mathrm{Fe}_{2} \mathrm{O}_{3}$ ), as shown in Fig. 3.

The intensity of the dip of the $\delta M$ plot depends strongly on the thickness of the nonmagnetic spacer shell. However, the variation of $\delta M$ with $t_{\mathrm{SiO}_{2}}$ clearly shows two regimes: 
strongly decreasing $\delta M$ for small shell thicknesses, and virtually constant for thick coatings. This behavior indicates that there is more than one mechanism involved, comprising dipolar interparticle interactions (dominating for small $t_{\mathrm{SiO}_{2}}$ ) and some intraparticle demagnetizing interactions present for all samples, but only evident when dipolar interactions are sufficiently weak. When comparing the shape of the $\delta M$ plots for strongly interacting VST0 and VSToa samples with the nominally non-interacting VST17 one (Fig. S2), it can be seen that while the non-interacting sample has a rather symmetric peak shape, the interacting samples show a considerably slower approach to zero at high fields (particularly visible when re-scaling the plots - see inset in Fig. S2a-), possibly indicating different mechanisms.

To assess the possible origin of the intraparticle interactions, we studied the low temperature hysteresis loops of the non-interacting samples in the series. As can be seen in Fig. S3, the field-cooled samples exhibit a sizable loop shift, $H_{E}$, along the field axis (i.e., exchange bias $\left.{ }^{90}\right)$. Interestingly, all the three samples with large $t_{\mathrm{SiO}_{2}}$ exhibit roughly the same $H_{E}$ $(\approx 100$ Oe $)$ value, consistent with the fact that they also show the same $\delta M$ dip. In fact, the existence of $H_{E}$ can be seen as an "intraparticle" deviation from the Wohlfarth's model for uniform monodomains with coherent switching. Notably, $H_{E}$ has often been observed in oxide ferrimagnets and it is frequently attributed to the presence of a thin highly-anisotropic spin-disordered layer at the surface of the nanoparticle. ${ }^{70,91}$ The presence of a spin-disordered layer is also revealed by a reduced "magnetic size" $7.2 \mathrm{~nm}$ (extracted from Langevin fits of room temperature loops, see Supporting Information) compared to the particle size evaluated from TEM images $d_{T E M}=8.0 \mathrm{~nm} .{ }^{79}$ Thus, this spin-disordered layer may be the origin of the unexpected $\delta M \operatorname{dip}$.

To test this hypothesis we studied the magnetic properties of the VCDx series, comprising nanoparticles with magnetic cores of different diameters coated with a thick silica shell magnetically isolating the cores. The top panels in Figure 4 present the size dependence of three different magnetic parameters: the depth of the $\mathrm{M}$ dip, the effective magnetic anisotropy constant, $K_{\text {eff }}$ (estimated from the blocking temperature, $K_{\text {eff }}=25 k T_{B} / V$ ) and 
the exchange bias field, $H_{E}$. Conveniently, the three types of particles with cores smaller than $8 \mathrm{~nm}\left(d_{T E M}=6.2,6.3,6.9 \mathrm{~nm}\right.$, corresponding to samples VCD6a, VCD6b and VCD7) display a zero $\delta M(H)$ curve [as shown in Fig. 2 (c)], as predicted by the Stoner-Wohlfarth model. Remarkably, these samples also show negligible exchange bias and a relatively low and constant $K_{\text {eff }}$. In contrast, for $d \geq 8 \mathrm{~nm}$ the particles exhibit strong $\delta M$ dips, similar to that shown in Fig. 2(b), as well as sizeable exchange bias fields and $K_{\text {eff }}$ values which vary with the reciprocal of the core diameter. This dependence (on the surface-to-volume ratio) suggests, indeed, the presence of a spin-disordered surface layer providing exchange bias and contributing to the effective anisotropy of the larger particles. The crossover region $(7-8 \mathrm{~nm})$ between these two magnetic regimes is highlighted by the grey shaded area in Fig. 4.

To investigate a possible structural origin for the different magnetic behavior in the two size regimes, the nanoparticle sizes obtained by TEM $\left(d_{T E M}\right)$ and x-ray diffraction $\left(\mathrm{XRD}, d_{X R D}\right)$ were compared. The results in Fig. $4(\mathrm{c})$ show that for small sizes $d_{X R D}$ is systematically larger than $d_{T E M}$, whereas the opposite holds for larger $(d \geq 8 \mathrm{~nm})$ sizes. That the XRD (Scherrer) sizes are somewhat larger than the TEM diameter in the case of the smaller $(d<8 \mathrm{~nm})$ nanoparticles can be understood from the volume-weighted character of the diffracted signal. The results in panel (c) (Fig. 4) imply that the smaller particles have a better crystallinity (the whole particle contributes coherently to the XRD) than the larger particles, where part of each particle (probably the near-surface region) is highly disordered and hence does not contribute to the diffraction peak profiles. These structural results correlate perfectly with the magnetic behavior described above, where the better crystallinity of the smaller particles strongly points to a lack of near-surface crystallographic disorder, which explains the absence of spin disorder related effects, i.e., the vanishing $\delta M$ dips and exchange bias fields together with the reduced $K_{\text {eff }}$ values with respect to the extrapolated (dashed) curve in Fig. 4(b). 


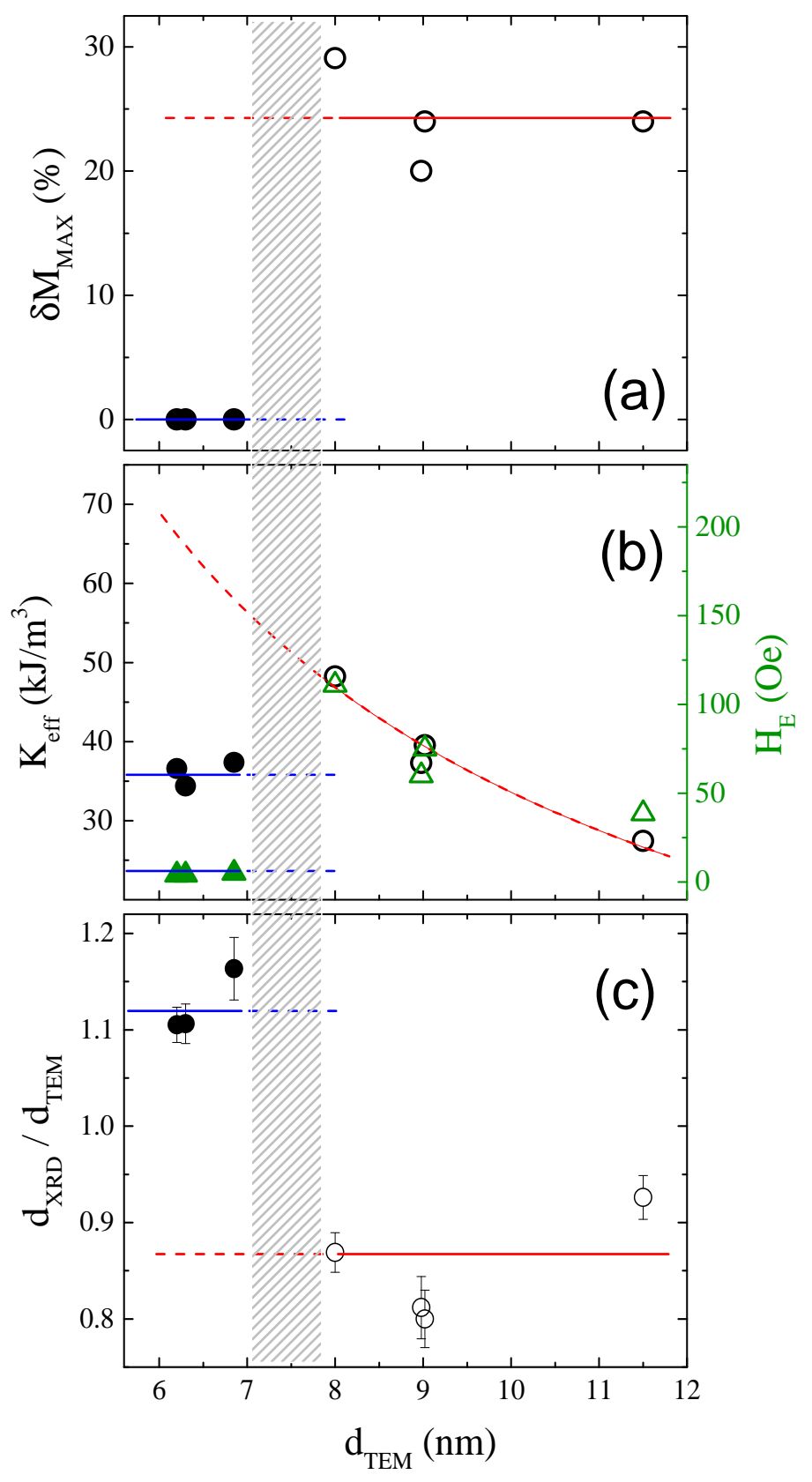

Figure 4: Particle size dependence of the depth of the $\delta M$ dip measured at $\mathrm{T}=5 \mathrm{~K}$ (a), the effective magnetic anisotropy constant (circles, left axis) and the exchange bias field (triangles, right axis) (b), and the ratio of the XRD and TEM nanoparticle sizes (c). The straight lines in the different panels are guides to the eye while the red curve in (b) is a fit to the inverse of the nanoparticle diameter $\left(1 / d_{T E M}\right)$, a dependence characteristic of surface or interface phenomena. The grey shaded area indicates the transition region between the two different behaviors. 


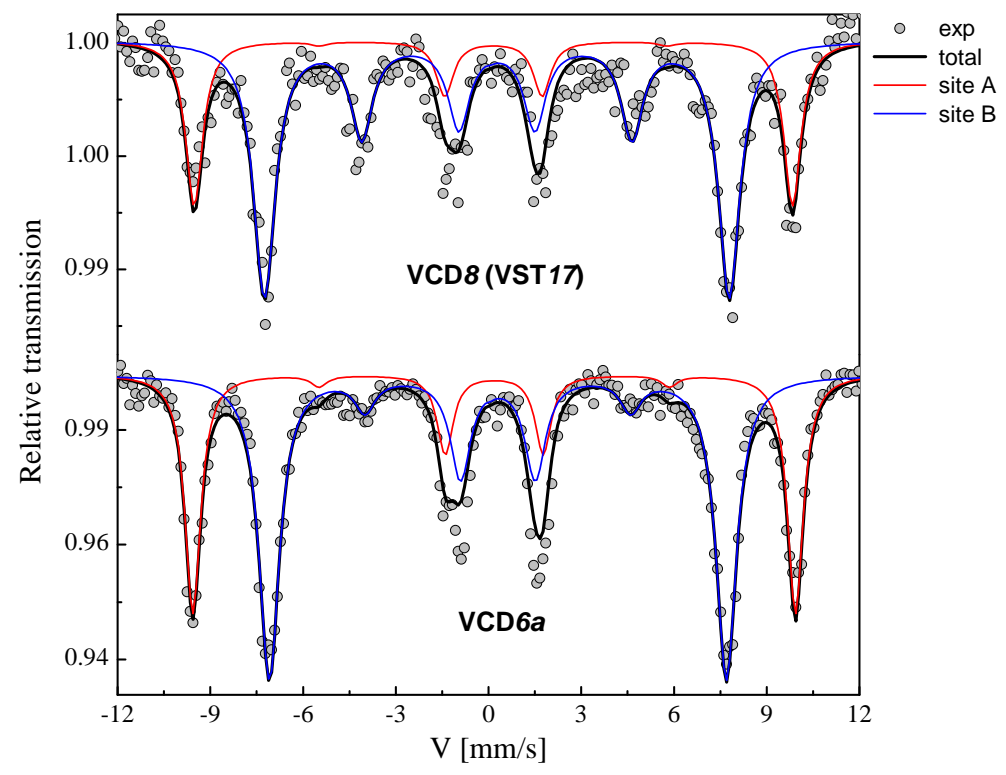

Figure 5: Mössbauer spectra obtained at $10 \mathrm{~K}$ in an $80 \mathrm{kOe}$ magnetic field applied parallel to the $\gamma$-beam in samples VCD8 (=VST17) and VCD6a. See Supporting Information for details of the Mössbauer fit.

\section{Mössbauer}

In order to firmly establish the presence of the crucial surface spin disorder indicated by the magnetic measurements, we have carried out an in-field Mössbauer study of two characteristic samples with thick $\mathrm{SiO}_{2}$ shells but very different magnetic behavior: VST17 (= VCD8, with sizable $\delta M$ and $H_{E}$ values) and VCD6a (with negligible $\delta M$ and $H_{E}$ ). As shown in Fig. 5, when comparing the high-field Mössbauer spectra of both samples, it is clear that the intensities of lines 2 and 5 strongly increase in sample VST17. This indicates that while most of the spins in VCD6a align with the magnetic field, in VST17 a fair amount of spins remain canted even at $80 \mathrm{kOe}^{92}$ (for a detailed discussion of the Mössbauer results, see Supporting Information ${ }^{91-95}$ ). This behavior is typical of nanoparticles with surface spin disorder. ${ }^{91,93}$ Using a core/shell model, ${ }^{95}$ a surface disordered shell of thickness about $0.8 \mathrm{~nm}$ is estimated, comparable to that obtained from Langevin estimates of the magnetic volume (see Supporting Information). Note that, in general, both volume and surface contributions may be present in Fe oxide particles. The former, related to frustrated topology, defects 
or oxidation gradients ${ }^{74,96-99}$ is unlikely in the present case given the high control of the oxidation process in the particle synthesis; however, it cannot be completely ruled out. Independently of the origin of the spin canting remaining in the $6 \mathrm{~nm}$ nanoparticles, it should be emphasized that it is rather small, equivalent to less than one surface atomic layer. Thus, the Mössbauer results fully confirm that the internal magnetic structure of the nanoparticles is the origin of the $\delta M$ features in samples without dipolar interactions.

\section{Monte Carlo simulations}

To corroborate the results we carried out a systematic Monte Carlo study of nanoparticles with a disordered surface layer. As it can be seen in Fig. 6(a), the simulation of single nanoparticles with such internal magnetic structure clearly shows a sizable $\delta M$ effect, in concordance with the experimental results, when the surface anisotropy is substantially larger than that of the core. The study of the diverse parameters (exchange, core anisotropy, surface anisotropy and thickness of the disordered layer) evidences that all the parameters are involved in the appearance of negative $\delta M$ (see Supporting Information). For example, the anisotropies $\left(K_{\text {Core }}\right.$ or $\left.K_{\text {Surface }}\right)$ have a non-monotonic effect on $\delta M$. As expected, for sufficiently small $K_{\text {Surface }}$ (e.g., $\left.K_{\text {Surface }}=0.1^{*} J_{F M}\right), \delta M$ virtually vanishes. On the other hand, increasing the thickness of the disordered surface layer leads to larger $\delta M$ values. Reducing the exchange causes a decrease in $\delta M$ in a complex fashion (with multiple peaks).

From the Monte Carlo simulations it can be inferred that the presence of a highlyanisotropic disordered surface layer leads to some locally antiparallel coupled spins. These spins make the magnetization process more difficult (i.e., higher fields are needed for the reversal), which translates into a "demagnetizing" dip in the $\delta M$ plot. The number of these antiparallel coupled spins (related, e.g., to the disordered surface thickness) and how they interact with each other (changes in $J, K_{\text {Core }}$ or $K_{\text {Surface }}$ ) dictate the strength of the effect.

This is further confirmed by the size effect on $\delta M$ [see Fig. 6(b)]. As $d$ decreases the role of the surface layer and thus of the antiparallel coupled spins becomes more prominent 

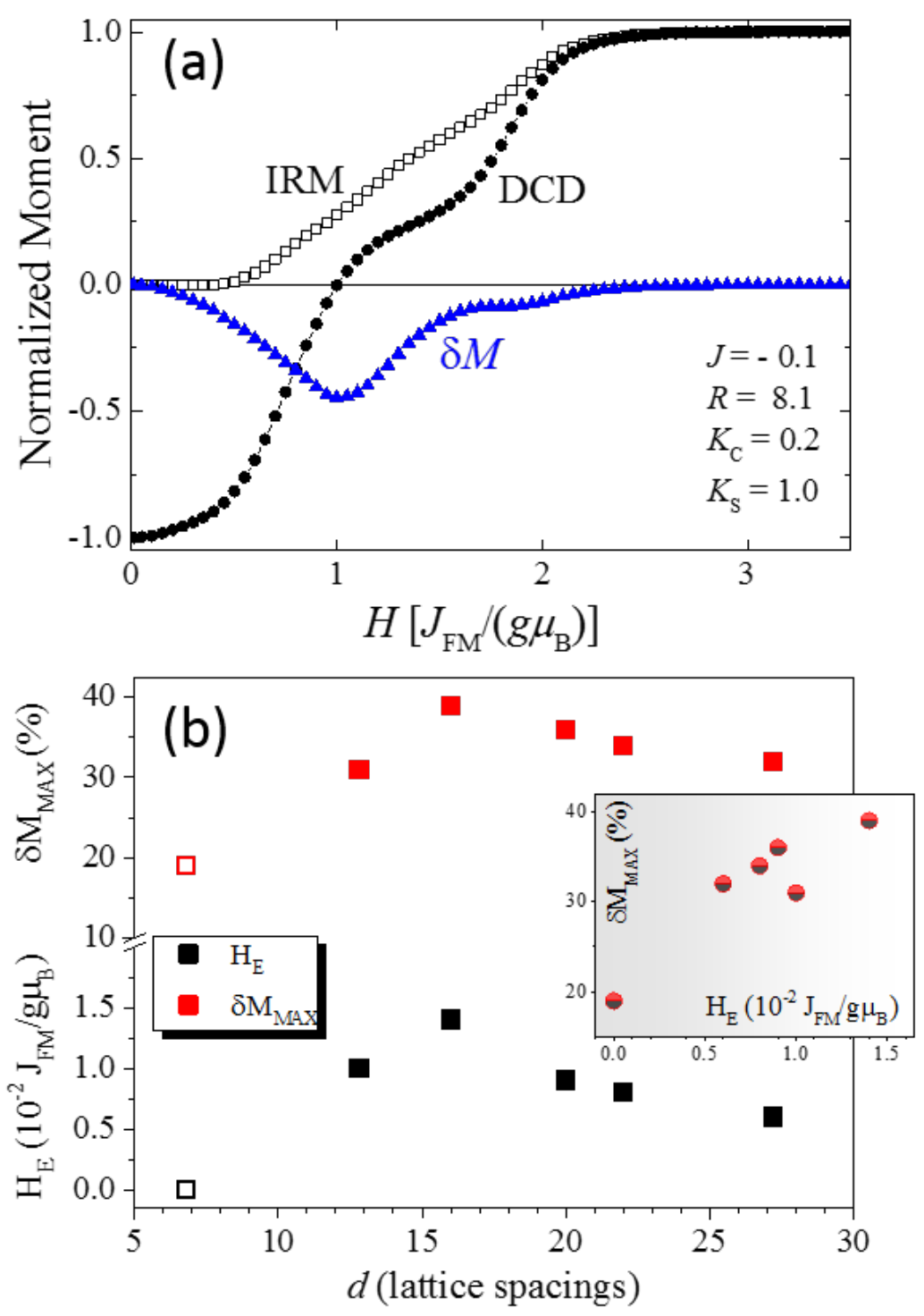

Figure 6: (a) Isothermal remanence, dc demagnetization remanence, and the resulting $\delta M$ plot for the indicated magnetic parameters. (b) Depth of the $\delta M$ dip and exchange bias field $H_{E}$ as a function of the particle diameter, $d$, and (inset) depth of the $\delta M$ dip as a function of exchange bias field $H_{E}$, for various particle sizes at low temperature.

and, consequently, $\delta M$ increases. For very small sizes, due to structural effects, ${ }^{79}$ the surface disorder becomes negligible, which leads to a decrease in $\delta M$. Remarkably, in full agreement with the experimental results, exchange bias in the simulations exhibits an analogous behavior to $\delta M$, as further confirmed by the correlation between $H_{E}$ and $\delta M$ shown in the inset in Fig. 6(b). Since in oxide ferrimagnetic nanoparticles $H_{E}$ is well-known to arise from 
the surface spin disorder, ${ }^{100}$ this implies that the negative $\delta M$ peak should also be correlated with the presence of surface disordered spins, although a simple one-to-one correlation between both effects cannot be concluded.

The above results demonstrate that using remanence plots to establish and quantify dipolar interactions in nanoparticle systems may be misleading, since systems with an absence of interparticle dipolar interactions but with an inhomogeneous internal magnetic structure (which does not behave as a simple macrospin) present clear non-zero $\delta M$ signals. More careful analysis of the shape of the $\delta M$ dips (see Supporting Information) indicates that when the dipolar interactions are strong the shape of the $\delta M$ is more asymmetrical and $\delta M$ goes to zero at much higher fields than when $\delta M$ arises from internal exchange interactions only. The measurement of a series of samples has allowed meaningful conclusions to be reached in the present study. However, it would be very difficult to ascertain the contribution of interparticle dipolar interactions to the $\delta M$ curve of a single particle assembly alone.

\section{First Order Reversal Curves}

The consistency of the above results justifies the use of our samples as benchmark systems to investigate the efficacy of other techniques in the search for reliable criteria to differentiate between the two effects leading to such similar $\delta M$ dips, namely intraparticle exchange and interparticle dipolar interactions. For instance, we have examined the magnetization switching of three representative samples using the comparatively sophisticated FORC analysis: (i) VCD6a - with essentially no dipolar interactions and no disordered magnetic layer, thus $\delta M=0$; (ii) VCD8 (VST17) - with essentially no dipolar interactions but with a disordered magnetic layer, thus with small but rather symmetric $\delta M(H)$, and (iii) VST0 with strong dipolar interactions and with a disordered magnetic layer, thus with large and asymmetric $\delta M(H)$. The families of FORCs and the corresponding FORC distributions for VCD6a, VCD8 (VST17) and VST0 are shown in Fig. 7. A continuous evolution is evident from weak interparticle interactions in the case of sample VCD6a, to stronger interactions 

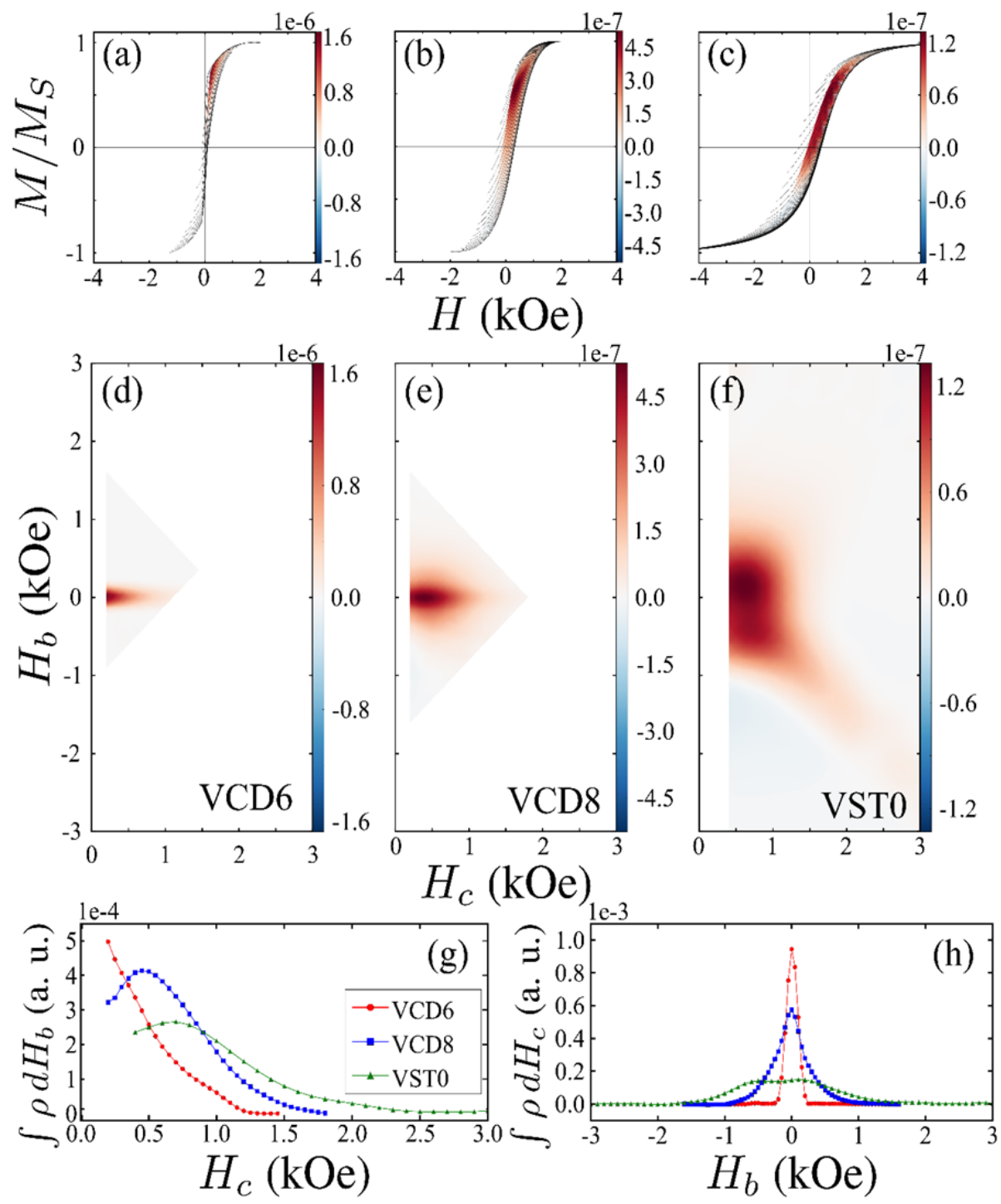

Figure 7: (a-c) Families of FORCs and (d-f) the corresponding FORC distributions for samples VCD6a, VCD8, and VST0, respectively. Color represents the height of the FORC distribution. (g-h) Projections of the FORC diagram onto $H_{c}$ and $H_{b}$ axes.

present in sample VCD8, and finally the highest level of interactions measured in sample VST0. The VCD6a sample exhibits a small coercivity of 110 Oe in the major loop [Fig. 7(a)]. The corresponding FORC distribution [Fig. 7(d)] exhibits a narrow ridge along the local coercivity $H_{c}$ axis, centered at $H_{c} \approx 200$ Oe, with no appreciable features along the $H_{b}$ direction, characteristic of non-interacting single domain magnetic nanoparticles. ${ }^{101,102}$ By comparison, the larger maghemite cores in VCD8 lead to a much-enhanced coercivity of 
330 Oe in the major loop [Fig. 7(b)], substantially larger than that in VCD6a. Similarly, the FORC distribution shifts to much larger local coercivity values, now centered at $H_{c} \approx$ 450 Oe [Fig. 7(e)]. This reflects the higher anisotropy due to the larger particle size as well as to the pinning of the magnetic core by the frozen moments at the surface/shell interface. These effects result in a larger spread and a decrease in the maximum of the switching field distribution, as can be observed in the FORC projections onto the $H_{c}$ axis [Fig. $\left.7(\mathrm{~g})\right]$. The FORC ridge is still largely centered along the $H_{c}$ axis, with limited spread along the $H_{b}$ axis, as illustrated by the projections of the FORC distributions along $H_{b}$ for each sample [Fig. 7(h)]. The qualitative similarities in both the FORC distributions and projections along $H_{b}$ for VCD6a and VCD8 indicate a similarly low degree of dipolar interactions, due to the large core-core separation. Sample VST0 exhibits again an enhanced major loop coercivity of 460 Oe [Fig. 7(c)]. The switching field distribution of VST0 has a lower maximum and a larger spread than those of VCD6a or VCD8, with the center of the FORC distribution located at $H_{c} \approx 700$ Oe [Figs. $7(\mathrm{f})$ and $\left.7(\mathrm{~g})\right]$. The FORC distribution is qualitatively different from those of VCD6a and VCD8, exhibiting a vertical ridge along the $H_{b}$ axis. Such a feature is characteristic of demagnetizing dipolar interactions, as seen in other systems, ${ }^{22,102,103}$ which are stronger in VST0 due to the much-reduced interparticle spacing than in either VCD6a or VCD8.

Strong dipolar interactions can be easily identified in a FORC diagram since they present additional features away from the central ridge along the $H_{c}$ axis ${ }^{104}$ [see Fig. $7(\mathrm{f})$ ]. However, when dipolar interactions are weak it may be difficult to discriminate between internal exchange interactions and dipolar interactions in the FORC diagrams. Nonetheless, close inspection of the projection along $H_{b}$ for VCD6a and VCD8 reveals an interesting feature, namely, the projection for VCD6a shows a more Gaussian-like shape, while that of VCD8 evidences a more Lorentzian-like character. Unfortunately, there are no systematic theoretical studies in nanoparticles to unambiguously assign the shape of the $H_{b}$ projections to a certain type of interactions. Hence, in this case FORC provides analogous information 
to that obtained form $\delta M$. More systematic experimental and theoretical work would be necessary to establish a correlation between these effects.

\section{Conclusions}

Inhomogeneous intraparticle magnetic structure has been clearly shown to cause the appearance of a strong $\delta M$ dip in isolated maghemite particles (for which TEM and magnetic behavior consistently confirm magnetic isolation of the maghemite cores by thick silica coating shells), very similar to the dips customarily attributed to dipolar interactions. The exchange coupling of a highly anisotropic layer of disordered surface spins (as confirmed by Mössbauer spectrometry) with a uniform ferrimagnetic core is the mechanism responsible for both exchange bias and the non-zero $\delta M$, as indicated by the correlation between the two parameters. The replication of the observed $\delta M$ dips in Monte Carlo simulations of isolated particles with an ordered core/disordered surface magnetic structure provides further support for the proposed intraparticle exchange mechanism. Our work thus strikes a cautionary note on the widespread use of remanence plots to assess interparticle/intergrain interactions, since effects due to complex internal magnetic structure may be easily overlooked, particularly in oxide particles, where surface spin disorder is commonly found. Conversely, our results demonstrate that, provided dipolar interactions can be safely ruled out by other means, $\delta M$ plots can be used to sensitively detect and possibly quantify intraparticle exchange coupling in magnetically non-uniform nanoparticles.

\section{Methods}

Two series of samples were prepared:

1. A varying-shell-thickness series (VSTx) comprising seven samples with a fixed $\gamma-\mathrm{Fe}_{2} \mathrm{O}_{3}$ core size $(8 \mathrm{~nm})$ coated with silica shells of varying thickness $\left(\mathrm{x}=t_{\mathrm{SiO}_{2}}=2,3,17,45\right.$ 
and $62 \mathrm{~nm})$. The series also includes nanoparticles coated with oleic acid $(\mathrm{x}=\mathrm{oa})$, as well as essentially bare particles $(\mathrm{x}=0)$ obtained after repeated washing in acetone of the oleic acid-coated nanoparticles. ${ }^{15}$ Figure 1 presents a schematic representation of this series, as well as transmission electron microscopy (TEM) images of the nanoparticles coated with thicker silica shells. ${ }^{15}$ In all cases the nanoparticles were pressed into disc-shaped pellets, the non-magnetic shell safely preventing any aggregation of the magnetic cores, controlling the distance between them and, therefore, the strength of dipolar interactions. The resulting maghemite concentration in the compacts (spanning almost four decades ${ }^{15}$ ) was determined by comparing the saturation magnetic moment in each disc with the saturation magnetization of the sample made of pure maghemite (VST0). These values are consistent with the volume fraction of magnetic material derived from the TEM images assuming a random close-packed configuration of the core/shell particles in the pressed discs.

2. A varying-core-diameter series (VCDx) comprising $\gamma-\mathrm{Fe}_{2} \mathrm{O}_{3}$ nanoparticles of varying diameter $d(\mathrm{x} \sim d=6.2,6.3,6.9,8.0,9.0$ and $11.5 \mathrm{~nm})$ coated by a thick $\mathrm{SiO}_{2}$ shell $\left(t_{\mathrm{SiO}_{2}} \approx 17 \mathrm{~nm}\right)$ in order to minimize dipolar interactions, ${ }^{79}$ and pressed into discs in the same fashion as the above series. Some of the samples were duplicated to confirm the reproducibility of the results. Under our sample nomenclature VCD8 and VST17 denote the same sample.

\section{Synthesis of maghemite nanoparticles}

Monodispersed $\gamma-\mathrm{Fe}_{2} \mathrm{O}_{3}$ nanoparticles were synthesized by thermal decomposition of $\mathrm{Fe}(\mathrm{CO})_{5}$ in the presence of an oleic acid stabilizer and dioctyl ether, followed by oxidation with trimethylamine N-oxide, $\left(\mathrm{CH}_{3}\right)_{3} \mathrm{NO} .{ }^{78}$ The size of the final nanoparticles was controlled by carefully adjusting the amount of oleic acid, while maintaining unaltered the other synthesis conditions, such as the volume of dioctyl ether and reaction temperature. For example, for the 11.5, 9, 8 and $6.2 \mathrm{~nm}$ nanoparticles, 3.0, 2.3, 2.0 and 1.5 mole equivalents of oleic acid 
were used, respectively. The reaction temperature was precisely controlled by a temperature controller with a thermocouple inserted in the reaction solution. For the synthesis of 11.5 nm nanoparticles, $3.84 \mathrm{~g}$ of oleic acid $(13.6 \mathrm{mmol})$ was put into a three-neck flask, followed by addition of $30 \mathrm{ml}$ of dioctyl ether. The solution was heated at $80{ }^{\circ} \mathrm{C}$ overnight with an appropriate flow of $\mathrm{Ar}$ or $\mathrm{N}_{2}$ to remove the remaining water in the solution. After injection of $0.6 \mathrm{ml}$ of $\mathrm{Fe}(\mathrm{CO})_{5}(4.56 \mathrm{mmol})$, the reaction solution was heated at $100{ }^{\circ} \mathrm{C}$ for $20 \mathrm{~min}$ and subsequently the reaction temperature was further increased to $300{ }^{\circ} \mathrm{C}$ at a rate of 4 ${ }^{\circ} \mathrm{C} / \mathrm{min}$ under Ar. The reaction solution was refluxed at $300{ }^{\circ} \mathrm{C}$ for $90 \mathrm{~min}$. Afterwards, the reaction solution was cooled down to $60{ }^{\circ} \mathrm{C}$ and $1.02 \mathrm{~g}$ of anhydrous $\left(\mathrm{CH}_{3}\right)_{3} \mathrm{NO}(13.6$ mmol) was added to the solution. Note that this compound is a mild oxidant which provides a highly controlled oxidation of the nanoparticles to $\gamma-\mathrm{Fe}_{2} \mathrm{O}_{3}$ (compared, e.g., to air). The resulting mixture was heated at $120{ }^{\circ} \mathrm{C}$ for $1 \mathrm{~h}$ and then heated to $290{ }^{\circ} \mathrm{C}$ at a ramping rate of $4{ }^{\circ} \mathrm{C} / \mathrm{min}$. The solution was heated at $290{ }^{\circ} \mathrm{C}$ for $1 \mathrm{~h}$. The resulting solution was cooled down and then acetone was added to precipitate the nanoparticles. The nanoparticles were collected by centrifugation. The nanoparticles can be dispersed in n-hexane and then precipitated again by adding acetone to remove the excess oleic acid. To obtain smaller maghemite nanoparticles, the above procedure was repeated by using a reduced amount of oleic acid. As the amount of oleic acid is decreased, the decomposition temperature of iron precursors decreases. The lower decomposition temperature probably leads to a larger number of seeds, yielding smaller nanoparticles.

\section{Silica coating of the maghemite nanoparticles}

For the silica coating of $11.5 \mathrm{~nm}$ maghemite nanoparticles (see below), $3.0 \mathrm{~g}$ of polyoxyethylene(5) nonylphenyl ether (6.80 mmol, Igepal CO-520) was dispersed in $16 \mathrm{ml}$ of cyclohex-

ane. ${ }^{105}$ Then, $350 \mu \mathrm{l}$ of $\gamma-\mathrm{Fe}_{2} \mathrm{O}_{3}$ solution $(28 \mathrm{mg} / \mathrm{ml}$ of cyclohexane) was added. The resulting mixture was sonicated, and $400 \mu \mathrm{l}$ of aqueous $\mathrm{NH}_{4} \mathrm{OH}(25 \mathrm{wt} \%$ ) was added under vigorous stirring and the final solution was additionally stirred for $1 \mathrm{~h}$ to yield a brown transpar- 
ent solution. An appropriate amount of tetraethyl orthosilicate (TEOS) was added to the solution and stirred for $24 \mathrm{~h}$. Starting from $100 \mu \mathrm{l}$ of TEOS, after checking the thickness of silica shell by TEM, the amount of TEOS was fine-tuned to yield the desired silica shell thickness. Finally, $5 \mathrm{ml}$ of methanol was added and vigorously stirred for $10 \mathrm{~min}$ to break the microemulsion. After stopping the stirring, the cyclohexane phase was discarded. $16 \mathrm{ml}$ of n-hexane was added to the methanol phase with silica-coated nanoparticles and vigorously stirred for $10 \mathrm{~min}$. Then the n-hexane phase was discarded and the silica-coated nanoparticles were collected by centrifuging the methanol phase. The obtained nanoparticles were thoroughly washed with methanol via repeated sonication and then centrifugation. The final nanoparticles were dried under high vacuum. Note that several steps were taken to avoid multiparticle coating. First, before the silica coating procedure, the ferrofluid solution was filtered through $450 \mathrm{~nm}$ filter to remove any larger aggregates of nanoparticles. To further minimize the number of possible smaller aggregates, $350 \mu \mathrm{l}$ of the ferrofluid solution was diluted to $1.5 \mathrm{ml}$ with cyclohexane and then sonicated for $30 \mathrm{~min}$. The diluted brown solution was centrifuged at 14,000 rpm for $1 \mathrm{~h}$ to yield a brown gradient solution. Then $1.3 \mathrm{ml}$ of the upper solution was taken and the rest (presumably containing aggregates) was discarded.

\section{Characterization}

Transmission electron microscopy (TEM) studies were carried out in a FEI Tecnai G2 F20 microscope operated at $200 \mathrm{kV}$. X-ray diffraction (XRD) measurements were carried out in transmission geometry using, for each maghemite particle size, just a few milligrams of the maghemite nanoparticles mounted on Kapton tape. A Bruker D8 diffractometer operating with $\mathrm{Cu} \mathrm{K}$ radiation and a position sensitive (silicon strip) detector was employed. From the most intense Bragg reflection of maghemite, (311), the XRD crystallite size was obtained by using the Scherrer equation for spherical particles, $d_{X R D}=(4 / 3) \lambda / \beta \cos \theta$, where $\lambda$, $\beta$ and $\theta$ are the XRD wavelength, the full width at half maximum of the reflection (in 
radians) and the Bragg angle, respectively. The magnetic measurements were performed using a Quantum Design MPMS Evercool SQUID magnetometer. The temperature (T) dependence of the magnetization was measured upon warming from $5 \mathrm{~K}$ in $H=5$ Oe after either zero-field-cooled (ZFC) or field-cooled (FC) protocols from room temperature.

Hysteresis loops were obtained at different temperatures after either ZFC or FC from room temperature to $5 \mathrm{~K}$ in $H=50 \mathrm{kOe}$. The $M_{r}(H)$ and $M_{d}(H)$ curves (see Supporting Information) were measured at $T=5 \mathrm{~K}$ (well below the blocking temperature of any of the studied particles) following thermal demagnetization [i.e., the initial demagnetized state prior to measuring $M_{r}(H)$ was achieved by cooling in zero field from the superparamagnetic regime]. From the $M_{d}(H)$ and $M_{r}(H)$ data, the $\delta M$ plots were computed.

Magnetization reversal characteristics of the samples were also studied using the FORC method. ${ }^{22,106-108}$ A strong magnetic field is applied to first saturate the sample. The field is then lowered to a specified reversal field $\mathrm{H}_{r}$, and the magnetization is measured as $H$ is increased back to saturation. A series of these measurements at various reversal fields makes up a complete set of FORCs. The FORC distribution is then determined using a mixed partial derivative, ${ }^{109}$

$$
\rho=-\frac{1}{2} \frac{\partial^{2} M\left(H, H_{r}\right)}{\partial H \partial H_{r}} .
$$

Alternatively, the FORC distribution can also be expressed in terms of the local coercivity $H_{c}=\left(H-H_{r}\right) / 2$ and bias $H_{b}=\left(H+H_{r}\right) / 2 .{ }^{110}$ Integration of $\rho\left(H, H_{r}\right)$ along $H_{b}$ and $H_{c}$ leads to the projection of the FORC distribution onto the $H_{c}$ and $H_{b}$ axis, respectively.

${ }^{57} \mathrm{Fe}$ Mössbauer spectra were recorded at $12 \mathrm{~K}$ in an $80 \mathrm{kOe}$ external field oriented parallel to the $\gamma$-ray from a ${ }^{57} \mathrm{Co} / \mathrm{Rh}$ source mounted on an electromagnetic drive, using a triangular velocity form, in a transmission scheme. The hyperfine structure was modelled by a leastsquare fitting procedure involving Zeeman sextets composed of Lorentzian lineshapes. The isomer shift values were referred to that of $\alpha$-Fe at $300 \mathrm{~K}$.

Simulations of the magnetic behavior of the nanoparticles were carried out using the Monte Carlo method with the implementation of the Metropolis algorithm. ${ }^{111}$ An initial 
simulation was carried out for a particle of $d=16.2$ lattice spacings (of which the surface comprises one lattice spacing) with $J=-0.1 * J_{F M}, K_{\text {Core }}=0.2 * J_{F M}$ and $K_{\text {Surface }}=$ $1.0 * J_{F M}$ (see Supporting Information for the details on the Monte Carlo simulations ${ }^{112,113}$ ). Based on this standard particle, several parameters have been systematically changed: (i) particle size (from 6 to 28 lattice spacings); (ii) surface thickness (up 2 lattice spacings); (iii) $\mathrm{J}\left(-0.1 * J_{F M}\right.$ and $\left.-0.3 * J_{F M}\right)$; (iv) $K_{C o r e}$ (from $0.1 * J_{F M}$ to $\left.0.6 * J_{F M}\right)$ and (v) $K_{\text {Surface }}$ (from $0.1 * J_{F M}$ to $\left.2.0 * J_{F M}\right)$. Based on the experimental result ${ }^{79}$ that for the smallest particles in the VCD series $(6.2 \mathrm{~nm})$ surface effects are not dominant, in the smallest simulated particles $(d=6.8$ lattice spacings $)$ the anisotropy strength of the disordered surface layer was taken to be equal to that of the core.

\section{Acknowledgement}

We thank E. Devlin and M. Hillenkamp for preliminary discussions, and M. Rivera and E. Prado for technical support. We acknowledge financial support from the Spanish MINECO (MAT2015-65295-R, MAT2016-77391-R), the Junta de Castilla-La Mancha (PEII-2014-042P), the Generalitat de Catalunya (2014-SGR-1015), the Brazilian agency CAPES (BEX 0298/2015-08), the Institute of Bioengineering and Nanotechnology (Biomedical Research Council, Agency for Science, Technology and Research, Singapore), the NCSRD-Siemens research project "Establishing a multidisciplinary and effective innovation and entrepreneurship hub" (E-11928) and the US NSF (DMR-1543582 and ECCS-1611424). ICN2 is founded by the CERCA Programme/Generalitat de Catalunya. ICN2 also acknowledges support from the Severo Ochoa Program (MINECO, Grant SEV-2013-0295). 


\section{ASSOCIATED CONTENT}

\section{Supporting Information}

Additional information on the $\delta M$ and the in-field Mössbauer techniques, table with the complete results of the Mössbauer spectra fits, details of the Monte Carlo simulations, FC and ZFC magnetization curves of the VST series (Fig. S1a), Langevin scaling of M(H;T) data measured in VST45 (Fig. S1b), details on the estimate of the "magnetic size" from Langevin fits, $\delta M$ plots of all the VST series and graphical analysis of the intraparticle and interparticle contributions to the dip (Fig. S2), example of hysteresis loops measured after ZFC and FC (for sample VST17, Fig. S3); X-ray diffraction patterns and lattice parameter across of the maghemite cores of different size (Fig. S4); complete results from Monte Carlo simulations showing the dependence of $\delta M$ on core anisotropy (Fig. S5), surface anisotropy (Fig. S6), exchange coupling constant (Fig. S7) and disordered surface thickness (Fig. S8). The Supporting Information is available free of charge.

\section{AUTHOR INFORMATION}

\section{Corresponding Author}

email: Josep.Nogues@uab.cat, JoseAngel.Toro@uclm.es

\section{Notes}

The authors declare no competing financial interest.

\section{References}

1. Chikazumi, S. Physics of Ferromagnetism; Oxford University Press, Oxford, 1997. 
2. Berger, A. Magnetization Reversal in Granular Thin Films. Physica B 2012, 40\%, $1322-1329$.

3. McCallum, R. W.; Lewis, L. H.; Skomski, R.; Kramer, M. J.; Anderson, I. E. Practical Aspects of Modern and Future Permanent Magnets. Annu. Rev. Mater. Res. 2014, 44, $451-477$.

4. Jiang, J. S.; Bader, S. D. Rational Design of the Exchange-Spring Permanent Magnet. J. Phys.: Condens. Matter 2014, 26, 064214.

5. O’Grady, K.; Laidler, H. The Limits to Magnetic Recording-Media Considerations. J. Magn. Magn. Mater. 1999, 200, 616-633.

6. Piramanayagam, S.; Srinivasan, K. Recording Media Research for Future Hard Disk Drives. J. Magn. Magn. Mater. 2009, 321, 485-494.

7. Wang, S.; Zhang, X.; Li, J.; Ma, J.; Huang, J. Enhanced Magnetic Softness and HighFrequency Characteristics of $\mathrm{Fe}_{51.1} \mathrm{Co}_{48.9} \mathrm{BAl}_{2} \mathrm{O}_{3}$ Nanogranular Films. Scripta Mater. 2011, $65,45-48$.

8. Krishnan, K. M. Biomedical Nanomagnetics: A Spin Through Possibilities in Imaging, Diagnostics, and Therapy. IEEE Trans. Magn. 2010, 46, 2523-2558.

9. Phenrat, T.; Saleh, N.; Sirk, K.; Tilton, R. D.; Lowry, G. V. Aggregation and sedimentation of aqueous nanoscale zerovalent iron dispersions. Environ. Sci. Technol. 2007, 41, 284-290.

10. Zhang, J.; Agramunt-Puig, S.; Del-Valle, N.; Navau, C.; Baró, M. D.; Estradé, S.; Peiró, F.; Pané, S.; Nelson, B. J.; Sanchez, A. et al. Tailoring Staircase-Like Hysteresis Loops in Electrodeposited Trisegmented Magnetic Nanowires: a Strategy toward Minimization of Interwire Interactions. ACS Appl. Mater. Interfaces 2016, 8, 4109-4117. 
11. Kostopoulou, A.; Lappas, A. Colloidal Magnetic Nanocrystal Clusters: Variable Length-Scale Interaction Mechanisms, Synergetic Functionalities and Technological Advantages. Nanotechnol. Rev. 2015, 4, 595-624.

12. Majetich, S.; Sachan, M. Magnetostatic Interactions in Magnetic Nanoparticle Assemblies: Energy, Time and Length Scales. J. Phys. D: Appl. Phys 2006, 39, R407-R422.

13. Huke, B.; Lücke, M. Magnetic Properties of Colloidal Suspensions of Interacting Magnetic Particles. Rep. Prog. Phys. 2004, 67, 1731-1768.

14. Martín, J.; Nogués, J.; Liu, K.; Vicent, J.; Schuller, I. K. Ordered Magnetic Nanostructures: Fabrication and Properties. J. Magn. Magn. Mater. 2003, 256, 449-501.

15. De Toro, J. A.; Normile, P. S.; Lee, S. S.; Salazar, D.; Cheong, J. L.; Muñiz, P.; Riveiro, J. M.; Hillenkamp, M.; Tournus, F.; Tamion, A. et al. Controlled Close-Packing of Ferrimagnetic Nanoparticles: an Assessment of the Role of Interparticle Superexchange versus Dipolar Interactions. J. Phys. Chem. C 2013, 117, 10213-10219.

16. Peddis, D.; Jönsson, P. E.; Varvaro, G.; Laureti, S. In Nanomagnetism: Fundamentals and Applications; Binns, C., Ed.; Elsevier B.V., Oxford, UK, 2014; pp 129-189.

17. Bitar, A.; Kaewsaneha, C.; Eissa, M. M.; Jamshaid, T.; Tangboriboonrat, P.; Polpanich, D.; Elaissari, A. Ferrofluids: from Preparation to Biomedical Applications. J. Colloid Sci. Biotechnol. 2014, 3, 3-18.

18. Bedanta, S.; Eimüller, T.; Kleemann, W.; Rhensius, J.; Stromberg, F.; Amaladass, E.; Cardoso, S.; Freitas, P. Overcoming the Dipolar Disorder in Dense CoFe Nanoparticle Ensembles: Superferromagnetism. Phys. Rev. Lett. 2007, 98, 176601.

19. Singh, G.; Chan, H.; Baskin, A.; Gelman, E.; Repnin, N.; Král, P.; Klajn, R. SelfAssembly of Magnetite Nanocubes into Helical Superstructures. Science 2014, 345, $1149-1153$. 
20. Disch, S.; Wetterskog, E.; Hermann, R. P.; Salazar-Alvarez, G.; Busch, P.; Brückel, T.; Bergström, L.; Kamali, S. Shape Induced Symmetry in Self-Assembled Mesocrystals of Iron Oxide Nanocubes. Nano Lett. 2011, 11, 1651-1656.

21. Heyderman, L. J.; Stamps, R. L. Artificial Ferroic Systems: Novel Functionality from Structure, Interactions and Dynamics. J. Phys.: Condens. Matter 2013, 25, 363201.

22. Gilbert, D. A.; Zimanyi, G. T.; Dumas, R. K.; Winklhofer, M.; Gomez, A.; Eibagi, N.; Vicent, J.; Liu, K. Quantitative decoding of interactions in tunable nanomagnet arrays using first order reversal curves. Sci. Rep 2014, 4, 4204.

23. López-Ortega, A.; Estrader, M.; Salazar-Alvarez, G.; Estradé, S.; Golosovsky, I. V.; Dumas, R. K.; Keavney, D. J.; Vasilakaki, M.; Trohidou, K. N.; Sort, J. et al. Strongly Exchange Coupled Inverse Ferrimagnetic Soft/Hard, $\mathrm{Mn}_{x} \mathrm{Fe}_{3 x} \mathrm{O}_{4} / \mathrm{Fe}_{x} \mathrm{Mn}_{3 x} \mathrm{O}_{4}$, Core/Shell Heterostructured Nanoparticles. Nanoscale 2012, 4, 5138-5147.

24. Löffler, J. F.; Braun, H.-B.; Wagner, W. Magnetic Correlations in Nanostructured Ferromagnets. Phys. Rev. Lett. 2000, 85, 1990-1993.

25. Krycka, K. L.; Borchers, J. A.; Laver, M.; Salazar-Alvarez, G.; López-Ortega, A.; Estrader, M.; Suriñach, S.; Baró, M.; Sort, J.; Nogués, J. Correlating MaterialSpecific Layers and Magnetic Distributions within Onion-Like $\mathrm{Fe}_{3} \mathrm{O}_{4} / \mathrm{MnO} / \gamma-\mathrm{Mn}_{2} \mathrm{O}_{3}$ Core/Shell Nanoparticles. J. Appl. Phys. 2013, 113, 17B531.

26. Sachan, M.; Bonnoit, C.; Majetich, S. A.; Ijiri, Y.; Mensah-Bonsu, P. O.; Borchers, J. A.; Rhyne, J. J. Field evolution of magnetic correlation lengths in -Co nanoparticle assemblies. Appl. Phys. Lett. 2008, 92, 152503.

27. Michels, A. Magnetic Small-angle Neutron Scattering of Bulk Ferromagnets. J. Phys.: Condens. Matter 2014, 26, 383201. 
28. Varón, M.; Beleggia, M.; Kasama, T.; Harrison, R.; Dunin-Borkowski, R.; Puntes, V. F.; Frandsen, C. Dipolar Magnetism in Ordered and Disordered Low-Dimensional Nanoparticle Assemblies. Sci. Rep. 2013, 3, 1234.

29. Yamamoto, K.; Majetich, S. A.; McCartney, M. R.; Sachan, M.; Yamamuro, S.; Hirayama, T. Direct Visualization of Dipolar Ferromagnetic Domain Structures in Co Nanoparticle Monolayers by Electron Holography. Appl. Phys. Lett. 2008, 93, 082502.

30. Puntes, V. F.; Gorostiza, P.; Aruguete, D. M.; Bastus, N. G.; Alivisatos, A. P. Collective Behaviour in Two-Dimensional Cobalt Nanoparticle Assemblies Observed by Magnetic Force Microscopy. Nat. Mater. 2004, 3, 263-268.

31. Palasantzas, G.; Koch, S. A.; Vystavel, T.; De Hosson, J. T. M. Nano-Sized Cobalt Cluster Films: Structure and Functionality. Adv. Eng. Mater. 2005, 7, 21-25.

32. Campanini, M.; Ciprian, R.; Bedogni, E.; Mega, A.; Chiesi, V.; Casoli, F.; de Julián Fernández, C.; Rotunno, E.; Rossi, F.; Secchi, A. et al. Lorentz Microscopy Sheds Light on the Role of Dipolar Interactions in Magnetic Hyperthermia. Nanoscale 2015, भ, 7717-7725.

33. Shimon, G.; Adeyeye, A. O. Direct Detection of Static Dipolar Interaction on a Single Nanodisk Using Microfocused Brillouin Light Scattering Spectroscopy. Adv. Electron. Mater. 2015, 1, 1500070.

34. Kortright, J. B.; Hellwig, O.; Chesnel, K.; Sun, S.; Fullerton, E. E. Interparticle Magnetic Correlations in Dense Co nanoparticle Assemblies. Phys. Rev. B 2005, 71, 012402.

35. Coey, J. M. D. Magnetism and Magnetic Materials; Cambridge University Press, 2009.

36. Kelly, P. E.; O'Grady, K.; Mayo, P. I.; Chantrell, R. W. Switching Mechanisms in Cobalt-Phosphorus Thin Films. IEEE Trans. Magn. 1989, 25, 3881-3883. 
37. Henkel, O. Remanenzverhalten und Wechselwirkungen in hartmagnetischen Teilchenkollektiven. Phys. Stat. Sol. (b) 1964, 7, 919-929.

38. Blanco-Andujar, C.; Ortega, D.; Southern, P.; Pankhurst, Q. A.; Thanh, N. T. K. High Performance Multi-Core Iron Oxide Nanoparticles for Magnetic Hyperthermia: Microwave Synthesis, and the Role of Core-to-Core Interactions. Nanoscale 2015, 7, $1768-1775$.

39. Coral, D. F.; Mendoza Zélis, P.; Marciello, M.; Morales, M. D. P.; Craievich, A.; Sánchez, F. H.; Fernández van Raap, M. B. Effect of Nanoclustering and Dipolar Interactions in Heat Generation for Magnetic Hyperthermia. Langmuir 2016, 32, 1201-1213.

40. Ojha, S.; Nunes, W. C.; Aimon, N. M.; Ross, C. A. Magnetostatic Interactions in SelfAssembled $\mathrm{Co}_{x} \mathrm{Ni}_{1-x} \mathrm{Fe}_{2} \mathrm{O}_{4} / \mathrm{BiFeO}_{3}$ Multiferroic Nanocomposites. ACS Nano 2016, 10, 7657-7664.

41. Nisar, M.; Bergmann, C.; Geshev, J.; Quijada, R.; Galland, G. B. An Efficient Approach to the Preparation of Polyethylene Magnetic Nanocomposites. Polymer 2016, 97, 131137.

42. Bohra, M.; Grammatikopoulos, P.; Diaz, R. E.; Singh, V.; Zhao, J.; Bobo, J.-F.; Kuronen, A.; Djurabekova, F.; Nordlund, K.; Sowwan, M. Surface Segregation in ChromiumDoped NiCr Alloy Nanoparticles and its Effect on their Magnetic Behavior. Chem. Mater. 2015, 27, 3216-3225.

43. Park, J.; Porter, M. D.; Granger, M. C. Silica Encapsulation of Ferrimagnetic Zinc Ferrite Nanocubes Enabled by Layer-By-Layer Polyelectrolyte Deposition. Langmuir 2015, 31, 3537-3545.

44. de la Vega, A. E.; Garza-Navarro, M. A.; Durán-Guerrero, J. G.; Cortez, I. E. M.; LucioPorto, R.; González-González, V. Tailoring the Magnetic Properties of Cobalt-Ferrite Nanoclusters. J. Nanopart. Res. 2016, 18, 18. 
45. Guerrero, A. L.; Mirabal-García, M.; Palomares-Sánchez, S. A.; Martínez, J. R. Effect of $\mathrm{Pb}$ on the Magnetic Interactions of the M-type Hexaferrites. J. Magn. Magn. Mater. 2016, 399, 41-45.

46. Nunes, W.; Cebollada, F.; Knobel, M.; Zanchet, D. Effects of Dipolar Interactions on the Magnetic Properties of $\gamma-\mathrm{Fe}_{2} \mathrm{O}_{3}$ Nanoparticles in the Blocked State. J. Appl. Phys. 2006, 99, 8N705.

47. Cao, C.; Tian, L.; Liu, Q.; Liu, W.; Chen, G.; Pan, Y. Magnetic Characterization of Noninteracting, Randomly Oriented, Nanometer-Scale Ferrimagnetic Particles. J. Geophys. Res.: Solid Earth 2010, 115, B07103.

48. Blanco-Mantecon, M.; O'Grady, K. Interaction and Size Effects in Magnetic Nanoparticles. J. Magn. Magn. Mater. 2006, 296, 124-133.

49. Peddis, D.; Cannas, C.; Musinu, A.; Piccaluga, G. Magnetism in Nanoparticles: Beyond the Effect of Particle Size. Chem. - Eur. J. 2009, 15, 7822-7829.

50. Cannas, C.; Musinu, A.; Ardu, A.; Orrù, F.; Peddis, D.; Casu, M.; Sanna, R.; Angius, F.; Diaz, G.; Piccaluga, G. $\mathrm{CoFe}_{2} \mathrm{O}_{4}$ and $\mathrm{CoFe}_{2} \mathrm{O}_{4} / \mathrm{SiO}_{2}$ Core/Shell Nanoparticles: Magnetic and Spectroscopic Study. Chem. Mater. 2010, 22, 3353-3361.

51. Dai, Q.; Lam, M.; Swanson, S.; Yu, R.-H. R.; Milliron, D. J.; Topuria, T.; Jubert, P.-O.; Nelson, A. Monodisperse Cobalt Ferrite Nanomagnets with Uniform Silica Coatings. Langmuir 2010, 26, 17546-17551.

52. Del Bianco, L.; Lesci, I.; Fracasso, G.; Barucca, G.; Spizzo, F.; Tamisari, M.; Scotti, R.; Ciocca, L. Synthesis of Nanogranular $\mathrm{Fe}_{3} \mathrm{O}_{4} /$ Biomimetic Hydroxyapatite for Potential Applications in Nanomedicine: Structural and Magnetic Characterization. Mater. Res. Express 2015, 2, 065002. 
53. Bottoni, G.; Candolfo, D.; Cecchetti, A.; Masoli, F. Evolution of the Interactions Effects with Co-Doping of Iron Oxide Particles. J. Magn. Magn. Mater. 1992, 104, 975-976.

54. Lakshmi, N.; Bhargava, H.; Suwalka, O.; Venugopalan, K.; Sebastian, V.; Reddy, V.; Gupta, A. Magnetic Properties Resulting from Core-Shell Interactions in Nanosized $\mathrm{Ni}_{0.25} \mathrm{Co}_{0.25} \mathrm{Zn}_{0.5} \mathrm{Fe}_{2} \mathrm{O}_{4}$. Phys. Rev. B 2009, 80, 174425 .

55. Li, H.; Li, X.; Guo, D.; Lou, L.; Li, W.; Zhang, X. Three-Dimensional Self-Assembly of Core/Shell-Like Nanostructures for High-Performance Nanocomposite Permanent Magnets. Nano. Lett. 2016, 16, 5631-5638.

56. Geshev, J.; Pereira, L. G.; Schmidt, J. E.; Mikhov, M. Dependence of the Magnetization and Remanence of Single-Domain Particles on the Second Cubic Anisotropy Constant. J. Appl. Phys. 2001, 90, 6243-6250.

57. Hillion, A.; Tamion, A.; Tournus, F.; Gaier, O.; Bonet, E.; Albin, C.; Dupuis, V. Advanced Magnetic Anisotropy Determination Through Isothermal Remanent Magnetization of Nanoparticles. Phys. Rev. B 2013, 88, 094419.

58. Blanco-Andujar, C.; Ortega, D.; Southern, P.; Pankhurst, Q. A.; Thanh, N. T. K. High Performance Multi-core Iron Oxide Nanoparticles for Magnetic Hyperthermia: Microwave Synthesis, and the role of Core-to-core Interactions. Nanoscale 2015, 7, $1768-1775$.

59. Dutz, S.; Andrä, W.; Hergt, R.; Müller, R.; Oestreich, C.; Schmidt, C.; Töpfer, J.; Zeisberger, M.; Bellemann, M. E. Influence of Dextran Coating on the Magnetic Behaviour of Iron Oxide Nanoparticles. J. Magn. Magn. Mater. 2007, 311, 51-54.

60. Coral, D. F.; Mendoza Zélis, P.; Marciello, M.; Morales, M. D. P.; Craievich, A.; Sánchez, F. H.; Fernández van Raap, M. B. Effect of Nanoclustering and Dipolar Interactions in Heat Generation for Magnetic Hyperthermia. Langmuir 2016, 32, 1201-1213. 
61. Blanco-Andujar, C.; Ortega, D.; Southern, P.; Pankhurst, Q. A.; Thanh, N. T. K. Nanoobjects for Addressing the Control of Nanoparticle Arrangement and Performance in Magnetic Hyperthermia. ACS Nano 2015, 9, 1408-1419.

62. Ferk, A.; Denton, J. S.; Leonhardt, R.; Tuffen, H.; Koch, S.; Hess, K.-U.; Dingwell, D. B. Paleointensity on Volcanic Glass of Varying Hydration States. Phys. Earth Planet. Inter. 2012, 208, 25-37.

63. Wehland, F.; Stancu, A.; Rochette, P.; Dekkers, M. J.; Appel, E. Experimental Evaluation of Magnetic Interaction in Pyrrhotite Bearing Samples. Phys. Earth Planet. Inter. 2005, 153, 181-190.

64. Fischer, H.; Mastrogiacomo, G.; Löffler, J. F.; J., W. R.; Weidler, P. G.; Gehring, A. U. Ferromagnetic Resonance and Magnetic Characteristics of Intact Magnetosome Chains in Magnetospirillum Gryphiswaldense. Earth Planet. Sci. Lett. 2008, 270, 200 - 208.

65. Proksch, R.; Moskowitz, B. Interactions between Single Domain Particles. J. Appl. Phys. 1994, 75, 5894-5896.

66. Wohlfarth, E. Relations between Different Modes of Acquisition of the Remanent Magnetization of Ferromagnetic Particles. J. Appl. Phys. 1958, 29, 595-596.

67. Bender, P.; Krämer, F.; Tschöpe, A.; Birringer, R. Influence of Dipolar Interactions on the Angular-Dependent Coercivity of Nickel Nanocylinders. J. Phys. D: Appl. Phys. 2015, 48, 145003 .

68. Geshev, J.; Mikhov, M. Remanence Curves for a Disordered System of Three- and FourAxial Fine Particles. Henkel-Type Plots. J. Magn. Magn. Mater. 1992, 104, 1569-1570.

69. Geshev, J.; Mikhov, M.; Schmidt, J. E. Remanent Magnetization Plots of Fine Particles with Competing Cubic and Uniaxial Anisotropies. J. Appl. Phys. 1999, 85, 7321-7327. 
70. Kodama, R. H.; Berkowitz, A. E.; McNiff Jr, E. J.; Foner, S. Surface Spin Disorder in $\mathrm{NiFe}_{2} \mathrm{O}_{4}$ Nanoparticles. Phys. Rev. Lett. 1996, 77, 394-397.

71. Krycka, K. L.; Booth, R. A.; Hogg, C. R.; Ijiri, Y.; Borchers, J. A.; Chen, W. C.; Watson, S. M.; Laver, M.; Gentile, T. R.; Dedon, L. R. et al. Core-Shell Magnetic Morphology of Structurally Uniform Magnetite Nanoparticles. Phys. Rev. Lett. 2010, 104, 207203.

72. Estrader, M.; López-Ortega, A.; Estradé, S.; Golosovsky, I. V.; Salazar-Alvarez, G.; Vasilakaki, M.; Trohidou, K. N.; Varela, M.; Stanley, D. C.; Sinko, M. et al. Robust Antiferromagnetic Coupling in Hard-Soft Bi-magnetic Core/Shell Nanoparticles. Nat. Commun. 2013, 4, 2960.

73. Peddis, D.; Yaacoub, N.; Ferretti, M.; Martinelli, A.; Piccaluga, G.; Musinu, A.; Cannas, C.; Navarra, G.; Greneche, J. M.; Fiorani, D. Cationic Distribution and Spin Canting in $\mathrm{CoFe}_{2} \mathrm{O}_{4}$ Nanoparticles. J. Phys.: Condens. Matter 2011, 23, 426004.

74. Disch, S.; Wetterskog, E.; Hermann, R. P.; Wiedenmann, A.; Vainio, U.; SalazarAlvarez, G.; Bergström, L.; Brückel, T. Quantitative Spatial Magnetization Distribution in Iron Oxide Nanocubes and Nanospheres by Polarized Small-angle Neutron Scattering. New J. Phys. 2012, 14, 013025.

75. Cichelero, R.; Harres, A.; Sossmeier, K. D.; Schmidt, J. E.; Geshev, J. Magnetic Interactions in Exchange-Coupled Yet Unbiased IrMn/NiCu Bilayers. J. Phys.: Condens. Matter 2013, 25, 426001 .

76. Muxworthy, A. R. Magnetic Hysteresis and Rotational Hysteresis Properties of Hydrothermally Grown Multidomain Magnetite. Geophys. J. Int. 2002, 149, 805-814.

77. Lavorato, G. C.; Peddis, D.; Lima Jr, E.; Troiani, H. E.; Agostinelli, E.; Fiorani, D.; Zysler, R. D.; Winkler, E. L. Magnetic Interactions and Energy Barrier Enhancement in Core/Shell Bimagnetic Nanoparticles. J. Phys. Chem. C 2015, 119, 15755-15762. 
78. Hyeon, T.; Lee, S. S.; Park, J.; Chung, Y.; Na, H. B. Synthesis of Highly Crystalline and Monodisperse Maghemite Nanocrystallites without a Size-Selection Process. J. Am. Chem. Soc. 2001, 123, 12798-12801.

79. Andersson, M. S.; Mathieu, R.; Lee, S. S.; Normile, P. S.; Singh, G.; Nordblad, P.; De Toro, J. A. Size-Dependent Surface Effects in Maghemite Nanoparticles and its Impact on Interparticle Interactions in Dense Assemblies. Nanotechnol. 2015, 26, 475703.

80. Stagi, L.; De Toro, J. A.; Ardu, A.; Cannas, C.; Casu, A.; Lee, S. S.; Ricci, P. C. Surface Effects Under Visible Irradiation and Heat Treatment on the Phase Stability of $\gamma$ - $\mathrm{Fe}_{2} \mathrm{O}_{3}$ Nanoparticles and $\gamma-\mathrm{Fe}_{2} \mathrm{O}_{3}-\mathrm{SiO}_{2}$ Core-Shell Nanostructures. J. Phys. Chem. C 2014, 118, 2857.

81. De Toro, J. A.; Lee, S. S.; Salazar, D.; Cheong, J. L.; Normile, P. S.; Muñiz, P.; Riveiro, J. M.; Hillenkamp, M.; Tournus, F.; Tamion, A. et al. A Nanoparticle Replica of the Spin-Glass State. Appl. Phys. Lett. 2013, 102, 183104.

82. Andersson, M. S.; De Toro, J. A.; Lee, S. S.; Mathieu, R.; Nordblad, P. Ageing Dynamics of a Superspin Glass. EPL 2014, 108, 17004.

83. Mathieu, R.; De Toro, J. A.; Salazar, D.; Lee, S. S.; Cheong, J. L.; Nordblad, P. Phase Transition in a Super Superspin Glass. EPL 2013, 102, 67002.

84. Franco, V.; Batlle, X.; Labarta, A.; O'Grady, K. The Nature of Magnetic Interactions in CoFe-Ag $(\mathrm{Cu})$ Granular Thin Films. J. Phys. D: Appl. Phys. 2000, 33, 609-613.

85. Álvarez, N.; Leva, E. S.; Valente, R.; Mansilla, M. V.; Gómez, J.; Milano, J.; Butera, A. Correlation between Magnetic Interactions and Domain Structure in A1 FePt Ferromagnetic Thin Films. J. Appl. Phys. 2014, 115, 083907.

86. Mørup, S.; Hansen, M. F.; Frandsen, C. Magnetic Interactions between Nanoparticles. Beilstein J. Nanotechnol. 2010, 1, 182-190. 
87. Lee, D. C.; Mikulec, F. V.; Pelaez, J. M.; Koo, B.; Korgel, B. A. Synthesis and Magnetic Properties of Silica-Coated FePt Nanocrystals. J. Phys. Chem. B 2006, 110, 1116011166.

88. Jing, L.; Yang, C.; Qiao, R.; Niu, M.; Du, M.; Wang, D.; Gao, M. Highly fluorescent CdTe $\mathrm{SiO}_{2}$ Particles Prepared via Reverse Microemulsion Method. Chem. Mater. 2010, 22, 420-427.

89. Allia, P.; Coisson, M.; Tiberto, P.; Vinai, F.; Knobel, M.; Novak, M. A.; Nunes, W. C. Granular Cu-Co alloys as Interacting Superparamagnets. Phys.Rev. B 2001, 64, 144420.

90. Nogués, J.; Sort, J.; Langlais, V.; Skumryev, V.; Suriñach, S.; Muñoz, J.; Baró, M. Exchange Bias in Nanostructures. Phys. Rep. 2005, 422, 65-117.

91. Salazar-Alvarez, G.; Qin, J.; Šepelák, V.; Bergmann, I.; Vasilakaki, M.; Trohidou, K. N.; Ardisson, J. D.; Macedo, W. A. A.; Mikhaylova, M.; Muhammed, M. et al. Cubic versus Spherical Magnetic Nanoparticles: The Role of Surface Anisotropy. J. Am. Chem. Soc. 2008, 130, 13234-13239.

92. Coey, J. M. D. Noncollinear Spin Arrangement in Ultrafine Ferrimagnetic Crystallites. Phys. Rev. Lett. 1971, 27, 1140-1142.

93. Peddis, D.; Cannas, C.; Piccaluga, G.; Agostinelli, E.; Fiorani, D. Spin-Glass-Like Freezing and Enhanced Magnetization in Ultra-Small $\mathrm{CoFe}_{2} \mathrm{O}_{4}$ Nanoparticles. Nanotechnol. 2010, 21, 125705.

94. Santoyo Salazar, J.; Perez, L.; de Abril, O.; Truong Phuoc, L.; Ihiawakrim, D.; Vazquez, M.; Greneche, J. M.; Begin-Colin, S.; Pourroy, G. Magnetic Iron Oxide Nanoparticles in 10-40 nm Range: Composition in Terms of Magnetite/Maghemite Ratio and Effect on the Magnetic Properties. Chem. Mater. 2011, 23, 1379-1386. 
95. Tronc, E.; Prené, P.; Jolivet, J. P.; Dormann, J. L.; Greneche, J. M. Spin Canting in $\gamma-\mathrm{Fe}_{2} \mathrm{O}_{3}$ Nanoparticles. Hyperfine Interact. 1998, 112, 97-100.

96. Wetterskog, E.; Tai, C.-W.; Grins, J.; Bergström, L.; Salazar-Alvarez, G. Anomalous Magnetic Properties of Nanoparticles Arising from Defect Structures: Topotaxial Oxidation of $\mathrm{Fe}_{1 x} \mathrm{O}-\mathrm{Fe}_{3 \delta} \mathrm{O}_{4}$ Core- Shell Nanocubes to Single-Phase Particles. ACS Nano 2013, 7, 7132-7144.

97. Baaziz, W.; Pichon, B. P.; Fleutot, S.; Liu, Y.; Lefevre, C.; Greneche, J.-M.; Toumi, M.; Mhiri, T.; Begin-Colin, S. Magnetic Iron Oxide Nanoparticles: Reproducible Tuning of the Size and Nanosized-Dependent Composition, Defects, and Spin Canting. J. Phys. Chem. C 2014, 118, 3795-3810.

98. Vichery, C.; Maurin, I.; Bonville, P.; Boilot, J.-P.; Gacoin, T. Influence of Protected Annealing on the Magnetic Properties of $\gamma-\mathrm{Fe}_{2} \mathrm{O}_{3}$ Nanoparticles. J. Phys. Chem. C 2012, 116, 16311-16318.

99. Morales, M. P.; Veintemillas-Verdaguer, S.; Montero, M. I.; Serna, C. J.; Roig, A.; Casas, L.; Martinez, B.; Sandiumenge, F. Surface and Internal Spin Canting in $\gamma-\mathrm{Fe}_{2} \mathrm{O}_{3}$ Nanoparticles. Chemistry of Materials 1999, 11, 3058-3064.

100. Mazo-Zuluaga, J.; Restrepo, J.; Muñoz, F.; Mejía-López, J. Surface Anisotropy, Hysteretic, and Magnetic Properties of Magnetite Nanoparticles: A Simulation Study. J. Appl. Phys. 2009, 105, 123907.

101. Cho, S.-J.; Shahin, A. M.; Long, G. J.; Davies, J. E.; Liu, K.; Grandjean, F.; Kauzlarich, S. M. Magnetic and Mössbauer Spectral Study of Core/Shell Structured Fe/Au Nanoparticles. Chem. Mater. 2006, 18, 960-967.

102. Pike, C. R.; Ross, C. A.; Scalettar, R. T.; Zimanyi, G. First-Order Reversal Curve Diagram Analysis of a Perpendicular Nickel Nanopillar Array. Phys. Rev. B 2005, 71, 134407. 
103. Kou, X.; Fan, X.; Dumas, R. K.; Lu, Q.; Zhang, Y.; Zhu, H.; Zhang, X.; Liu, K.; Xiao, J. Q. Memory Effect in Magnetic Nanowire Arrays. Adv. Mater. 2011, 23, 13931397.

104. Gilbert, D. A.; Liao, J. W.; Kirby B J, M., Winklhofer; Lai, C. H.; Liu, K. Magnetic Yoking and Tunable Interactions in FePt-Based Hard/Soft Bilayers. Sci. Rep. 2016, 6, 32842.

105. Yi, D. K.; Lee, S. S.; Papaefthymiou, G. C.; Ying, J. Y. Nanoparticle Architectures Templated by $\mathrm{SiO}_{2} / \mathrm{Fe}_{2} \mathrm{O}_{3}$ Nanocomposites. Chem. Mater. 2006, 18, 614-619.

106. Pike, C. R.; Roberts, A. P.; Verosub, K. L. Characterizing Interactions in Fine Magnetic Particle Systems using First Order Reversal Curves. J. Appl. Phys. 1999, 85, 66606667.

107. Davies, J. E.; Hellwig, O.; Fullerton, E. E.; Denbeaux, G.; Kortright, J. B.; Liu, K. Magnetization Reversal of CoPt Multilayers: Microscopic Origin of High-Field Magnetic Irreversibility. Phys. Rev. B 2004, 70, 224434.

108. Gilbert, D. A.; Maranville, B. B.; Balk, A. L.; Kirby, B. J.; Fischer, P.; Pierce, D. T.; Unguris, J.; Borchers, J. A.; Liu, K. Realization of Ground-State Artificial Skyrmion Lattices at Room Temperature. Nat. Commun. 2015, 6, 8462.

109. Mayergoyz, I. D. Mathematical Models of Hysteresis; Springer-Verlag, 1991.

110. Dumas, R. K.; Liu, K.; Li, C.-P.; Roshchin, I. V.; Schuller, I. K. Temperature Induced Single Domain-Vortex State Transition in sub-100 nm Fe Nanodots. Appl. Phys. Lett. 2007, 91, 202501.

111. Vasilakaki, M.; Eftaxias, E.; Trohidou, K. N. Monte Carlo Study of the Exchange Bias and the Training Effect in Nanoparticles with Core/Shell Morphology. Phys. Stat. Solid. (a) 2008, 205, 1865-1871. 
112. Bødker, F.; Mørup, S.; Linderoth, S. Surface Effects in Metallic Iron Nanoparticles. Phys. Rev. Lett. 1994, 72, 282-285.

113. Dimitrov, D. A.; Wysin, G. M. Magnetic Properties of Superparamagnetic Particles by a Monte Carlo Method. Phys. Rev. B 1996, 54, 9237-9241. 